\title{
Una visita contra el estigma y por la prevención en un centro de tratamiento para trastorno mental grave
}

\author{
María CASTILlo LASALA ${ }^{1}$ \\ castillolasalamaria@gmail.com \\ Luis ORNA DÍAZ ${ }^{2}$ \\ romanopatroni@hotmail.com \\ José Antonio PÉREZ ROJO ${ }^{3}$ \\ joseanrojo@gmail.com
}

Recibido: $15 / 03 / 12$

Aceptado: 22/11/12

\section{RESUMEN}

Uno de los ejes principales de trabajo en nuestra unidad rehabilitadora para el tratamiento del trastorno mental grave es la lucha contra la lacra del estigma. Como ejemplo de nuestro quehacer diario relatamos la visita de una escuela de auxiliares de enfermería a nuestra unidad. La visita se convirtió en una experiencia de relación entre visitantes, usuarios y personal del centro en la que mediante juegos, actividades de expresión escrita y corporal se trabajaron conceptos como el estigma y la prevención. La participación activa de los usuarios en todas las actividades y su implicación emocional con los visitantes les permitieron avanzar en su rehabilitación y su inclusión social, superando el estigma.

Palabras clave: trastorno mental grave, estigma, prevención, rehabilitación, inclusión social

\section{Referencia normalizada}

Castillo Lasala, M., Orna Diaz, L., Pérez Rojo, J. A. (2012). "Una visita contra el estigma y por la prevención en un centro de tratamiento para trastorno mental grave". En Arteterapia: Papeles de arteterapia y educación artística para la inclusión social Vol.7: páginas 281-294. Madrid. Servicio de publicaciones UCM.

\footnotetext{
${ }^{1}$ Psicóloga clínica, con una experiencia de 30 años en el tratamiento del trastorno mental grave. Coordinadora de la UME de Guadalajara desde hace 5 años. Unidad de Media Estancia (UME) de Guadalajara, Servicio de Salud Mental de Guadalajara. SESCAM, Calle Hospital s/n 19001 Guadalajara

${ }^{2}$ Terapeuta ocupacional con formación en el campo del arte dramático, ha trabajado durante años con el trastorno mental grave. Unidad de Media Estancia (UME) de Guadalajara, Servicio de Salud Mental de Guadalajara. SESCAM, Calle Hospital s/n 19001 Guadalajara

${ }^{3}$ Psiquiatra, con una experiencia de 10 años en el tratamiento del trastorno mental grave en centros rehabilitadores. Unidad de Media Estancia (UME) de Guadalajara, Servicio de Salud Mental de Guadalajara. SESCAM, Calle Hospital s/n 19001 Guadalajara
} 


\title{
SUMARIO
}

Introducción. La Unidad de Media Estancia (UME). La experiencia. Primera dinámica: un ejercicio revelador de la fragilidad de nuestros prejuicios. Unas notas acerca del estigma Segunda dinámica: la enfermedad mental y la salud mental. Tercera dinámica: una dinámica corporal de todo el grupo. Cuarta dinámica: una sesión de psicoeducación por parte de los usuarios. Conclusiones y reflexiones. Bibliografía

\section{A visit against stigma and pro prevention in a treatment unit for serious men-} tal illness

\begin{abstract}
One of the main goals of our rehabilitation unit for the serious mental illness is the fight against the scourge of stigma. As an example of our daily work we review the visit that a school of practical nurse students made us. The visit became a relational experience between the visitors and the users of the unit in which through games, oral expression and body language activities we worked with concepts as stigma and prevention. The active participation of the users in the activities and their emotional implication with the visitors made them move forward in their rehabilitation and social inclusion overcoming stigma..
\end{abstract}

Keywords: serious mental illness, stigma, prevention, rehabilitation, social inclusion

\section{CONTENTS}

Introduction. Media Stay Unit (EMU). Experience. First dynamics: an exercise reveals the fragility of our prejudices. Some notes about stigma Second dynamics: mental illness and mental health. Third dynamic: a dynamic body of the whole group. Fourth dynamics: a session psychoeducation by users. Conclusions and reflections. Bibliography

\section{INTRODUCCIÓN}

La visita a la Unidad de Media Estancia para Trastorno Mental Grave (UME) de Guadalajara de los estudiantes del grado formativo de Técnico en Cuidados Auxiliares de Enfermería de la Escuela Familiar Agraria (EFA) "El Llano" de Humanes de Mohernando, fue el comienzo de esta experiencia. Su programa formativo incluye visitas a diversos centros, para poder observar de forma directa, el trabajo del auxiliar de enfermería.

En la UME decidimos aprovechar la ocasión para que nuestros visitantes, treinta alumnos de entre 18 y 50 años, pudieran conocer la realidad del enfermo mental y trabajar con el estigma que recae sobre las personas con Trastorno Mental Grave (TMG).

Para ello, partiendo de la psicoeducación y la terapia ocupacional, disciplinas que empleamos diariamente en nuestra unidad, generamos una serie de dinámicas en las que a través del descubrimiento, el juego y el cuerpo fuésemos modificando las creencias, los prejuicios, la discriminación en relación con los enfermos mentales.

\section{LA UNIDAD DE MEDIA ESTANCIA (UME)}

La UME es un dispositivo sanitario y rehabilitador, de puertas abiertas, que proporciona tratamiento integral dirigido a personas con trastorno mental grave y a sus 
familias. Está situada en el centro de la ciudad de Guadalajara, en un edificio de nueva construcción rodeado de jardines, bien comunicado con la ciudad. La capacidad actual es de 12 pacientes que ingresan derivados desde la Unidad de Salud Mental (USM) y desde la Unidad de Hospitalización Breve (UHB) del Hospital Universitario de Guadalajara. Se trata de pacientes psiquiátricos graves, con una evolución complicada. Cuando son derivados a la UME, generalmente han fracasado los tratamientos ambulatorios y están abocados a un aislamiento casi total y a múltiples reingresos psiquiátricos.

En la UME trabaja un equipo interdisciplinar integrado por distintos profesionales (psicóloga, psiquiatra, terapeuta ocupacional, médico general, trabajadora social, enfermeros y auxiliares), que desde sus diferentes áreas están involucrados activamente en un proyecto de rehabilitación biopsicosocial para el paciente cuya estancia media en la unidad suele ser de entre 6 y 12 meses. Los objetivos de la unidad son el control de la patología psiquiátrica, la recuperación del nivel funcional, la adquisición o recuperación de habilidades y recursos psicológicos, sociales y laborales para favorecer la integración en su núcleo familiar y social. Los programas de rehabilitación más importantes con los que se trabaja son: psicoterapia individual y grupal, psicoeducación y estilos de vida saludables, habilidades sociales, intervenciones familiares y grupo de familias, rehabilitación cognitiva, psicomotricidad, actividades de la vida diaria, educación para adultos, club de lectura, programa de ocio y tiempo libre y reinserción laboral. Además, desde hace casi una década, contamos con un programa de arteterapia en colaboración con el Máster de Arteterapia y Educación Artística para la Inclusión Social de las Universidades Complutense y Autónoma de Madrid, cuyos alumnos realizan prácticas en el centro.

\section{LA EXPERIENCIA}

La visita de los alumnos de Cuidados Auxiliares de Enfermería de la Escuela "El Llano", la tomamos, en el equipo de la UME, como una oportunidad que nos permitía plantearnos preparar una jornada que sirviera tanto a los visitantes como a los integrantes de la UME como espacio psicoeducativo en relación con la enfermedad y la salud mental.

\section{PRIMERA DINÁMICA: UN EJERCICIO REVELADOR DE LA FRAGILI- DAD DE NUESTROS PREJUICIOS}

Jugando a las adivinanzas, ¿Quién soy yo? ¿Quién eres tú?

Cuando se entra en un establo es fácil discriminar quién es oveja y quién es pastor...

En una residencia de ancianos podemos diferenciar al celador y al cliente...

En el caso de personas con enfermedad mental no es tan fácil, a los ojos de un visitante.

Más allá de la locura personal de cada humano, los prejuicios de nuestra sociedad, -que son los nuestros, los de todos y todas-, tienden a asociar una serie de 
características de imagen y de comportamiento para clasificar a la persona "loca"; con toda la carga que el estigma conlleva.

Con hospitalidad, en sus dos acepciones, (Hospitalidad: 1.Buen recibimiento que se hace a los visitantes. 2. Estancia de los enfermos en el hospital), hicimos de anfitriones a un nutrido grupo de estudiantes.

Nos proponíamos abrir un hueco en los prejuicios sociales, desmarcarnos de "estos son ellos y estos somos nosotros" y queríamos hacerlo en un marco lúdico, de descubrimiento. Así que nos presentamos todos como personas, sin apellidos y sin etiquetas. Y así empezó la visita, con un juego. Todos por parejas, en cada par un anfitrión y un visitante. Todos y todas jugando a adivinar quién es qué y a que nos dedicamos cada cual.

Los estudiantes y sus profesoras entraron a una sala donde se encontraron a unas veinte personas, terapeutas y usuarios de la UME. Todos íbamos vestidos de calle de modo que por nuestro atuendo no pudiera distinguirse quién era usuario y quién era terapeuta; por cierto, algo habitual en nuestra unidad. Nos sentamos todos haciendo un gran círculo en la sala común.

El ejercicio propuesto consistió en que cada visitante se emparejase con alguien de la UME al azar. Cada persona debería hacer tres o cuatro preguntas al desconocido que le hubiese tocado que le permitieran hacerse idea de quién era, para posteriormente presentarlo. Estaba permitido preguntar cualquier cosa menos la profesión, qué hacíamos en la UME, o sobre la enfermedad. Las preguntas las hacíamos tanto los visitantes como todos los integrantes de la UME y transcurridos unos minutos realizamos una ronda de presentación.

Algunos de los pacientes fueron presentados como terapeutas, un paciente que acababa de ingresar y que no se encontraba precisamente asintomático fue identificado como el psiquiatra de la unidad, mientras que al psiquiatra y al médico de admisión del centro los identificaron como pacientes, así como al terapeuta ocupacional. Por ejemplo, a la coordinadora de la unidad se la identificó como la secretaria. Nuestros visitantes estaban confusos, sorprendidos. Alguno pasó mucho rato disculpándose por habernos identificado como pacientes.

Nosotros habíamos pensado en este ejercicio como una herramienta para cuestionar el mito de que "a los enfermos mentales se les reconoce por su aspecto" y el mito había caído estrepitosamente.

\section{UNAS NOTAS ACERCA DEL ESTIGMA}

Estigma" es un término con el que hacemos referencia a un conjunto de actitudes, habitualmente negativas, que un grupo social mantiene con otros grupos minoritarios, en virtud de que éstos presentan algún tipo de rasgo diferencial o "marca" que permite identificarlos ${ }^{4}$. En el estigma se asocian rasgos simples, fácilmente identificables como rasgos físicos, aspecto, conductas, etc., con tipos de personas que pue-

\footnotetext{
${ }^{4}$ GOFMAN E. Estigma. La identidad deteriorada. Buenos Aires: Ed. Amorrortu. 1970.
} 
den considerarse potencialmente peligrosas: extranjeros, enfermos, delincuentes, etc. A la enfermedad mental se asocian en el imaginario colectivo: criminalidad, accidentes de tráfico, abusos y violencia doméstica y de género, vagabundeo, etc. Sin embargo, sabemos que las personas con trastornos mentales contribuyen poco a la generación de estas lacras sociales.

La Asociación Americana de Psiquiatría (APA), defiende que, "la mayor parte de las personas violentas no sufren enfermedad mental y que con tratamiento, la persona con trastorno mental no es más peligrosa que la población general". Los investigadores niegan la asociación entre enfermedad mental y violencia, no encuentran diferencias significativas en la frecuencia de actos criminales entre población general y personas con trastorno esquizofrénico. En hospitales de Estocolmo en 1971 se realizó un amplio estudio sobre 644 pacientes esquizofrénicos en seguimiento durante el periodo 1972-86 y se encontró que los enfermos esquizofrénicos no cometieron más delitos que los esperados en la población general.5 Otros estudios coinciden en la opinión de que las personas con enfermedad mental no son más violentas que la población general cuando se tienen en cuenta factores extrínsecos como las variables demográficas.

Los medios de comunicación tratan la enfermedad mental asociándola a la violencia. Utilizan significaciones inadecuadas que, aunque contradicen las opiniones de profesionales expertos, ahondan en el proceso de estigmatización.6 La mayor parte de los enfermos diagnosticados de trastorno esquizofrénico no muestran agresividad patológica y estudios recientes han encontrado asociación entre violencia y esquizofrenia únicamente en aquellos pacientes que se da como circunstancia añadida el abuso de drogas y el incumplimiento terapéutico. ${ }^{7}$

Todos estos factores pueden reducirse de manera muy considerable mediante la puesta en marcha de programas específicos para el tratamiento de los pacientes esquizofrénicos que incrementen la conciencia de enfermedad, controlen los síntomas, prevengan la aparición de recaídas y transmitan al paciente la necesidad de un correcto cumplimiento de la medicación. En suma, un tratamiento adecuado es el mejor elemento de prevención para la conducta violenta en la esquizofrenia.

En el caso del enfermo mental se identifica completamente a la persona con la patología. Se lanzan sobre ella todos los prejuicios generados por falsos mitos. El estigma social es una carga añadida de angustia y desamparo. Desde la mirada que estigmatiza, la enfermedad mental no es "algo que alguien tiene", sino "algo que alguien es". Cada vez nos extrañaría mas que alguien llamara "canceroso" a al-

\footnotetext{
${ }^{5}$ LINQVIST P, ALLEBECK P. Schizophrenia and crime: a longitudinal follow-up of 644 shizophrenics in Stockholm. British Journal of Psychiatry 1990; 157:345-350.

${ }^{6}$ LÓPEZ M. y LAVIANA M. La lucha contra el estigma y la discriminación en salud mental. Una estrategia compleja basada en la información disponible Rev. Asoc. Esp. Neuropsiq. 2008 v. 28 n. 1 Madrid.

${ }^{7}$ GONZALEZ PARRA S, MOLINA JD, HUERTAS D. Violencia asociada con los trastornos esquizofrénicos. Archivos de Psiquiatría 2008, Núm. 2-8 Vol. 71-p. 109-127.
} 
guien que padece un cáncer, (utilizando el adjetivo como genérico) sin embargo nos parece normal llamar "esquizofrénico" a alguien que padece esquizofrenia.

Los componentes cognitivos, emocionales y conductuales en el estigma van a dar lugar a estereotipos, prejuicios y discriminación. El estereotipo es el conjunto de creencias, en gran parte erróneas, que la mayoría de la población mantiene en relación con un determinado grupo social y que sesgan en gran medida la percepción, el recuerdo y la valoración de muchas de las características y conductas de los miembros de dicho grupo. Creencias negativas que se asocian habitualmente con el colectivo de la enfermedad mental son peligrosidad, incompetencia, falta de voluntad. En cuanto a los prejuicios, son predisposiciones emocionales, habitualmente negativas, que la mayoría experimenta con respecto a los miembros del grupo cuyas características están sujetas a creencias estereotipadas. La conformidad con estas creencias y prejuicios da lugar a reacciones emocionales habitualmente negativas como el miedo. Y por último, como consecuencia de todo esto se encuentra la discriminación, que es la propensión a desarrollar acciones positivas o negativas, habitualmente medidas en términos de distancia social deseada, hacia los miembros sobre los que recae el estigma. ${ }^{8}$ Dentro de la esfera de la discriminación encontramos comportamientos en respuesta al prejuicio como rechazo, negativa a emplear o alojar, negativa a ayudar, etc. ${ }^{9}$

El autoestigma es la internalización de ideas y reacciones en quienes sufren la estigmatización. Los propios enfermos mentales en muchos casos desarrollan creencias negativas sobre sí mismos. La asociación entre estigmatización percibida y autoestigma es relativamente elevada en la población de pacientes esquizofrénicos. En los estudios, los resultados indican que el aislamiento social tiene una relación significativa con el autoestigma que es el aspecto que mejor predice el nivel de autoestima.6

En la formación del estigma intervienen factores como la generalización y categorización de las personas, el menosprecio de aquello que es diferente, la aceptación acrítica de estereotipos y el desconocimiento por escasez de información y sensibilidad. No debemos olvidar que también influye la discriminación administrativa sobre aspectos rehabilitadores y laborales.

Es por eso, que es fundamental tomar iniciativas que aumenten el conocimiento de la enfermedad mental y ayuden a reducir el estigma y la marginación asociados a las personas que sufren estos trastornos y a sus familiares.

Tras la primera dinámica de grupo quedamos sorprendidos y satisfechos, había caído un pilar del estigma: a los enfermos mentales no se les distingue por la calle, ni siquiera a los enfermos mentales graves.9

${ }^{8}$ CORRIGAN P W, WATSON AC. Understanding the Impact of Stigma on People with Mental Illness. World Psychiatry, 2002, 1, 1, pp. 16-20.

${ }^{9}$ LAVIANA M. La atención a las personas con Esquizofrenia y otros trastornos mentales graves desde los servicios públicos: una atención integral e integrada en un modelo comunitario. Apuntes de Psicología 2006, 24, 1-3, pp. 345-373. 


\section{SEGUNDA DINÁMICA: LA ENFERMEDAD MENTAL Y LA SALUD MENTAL}

El segundo ejercicio que hicimos con el grupo fue una breve sesión psicoeducativa alrededor de conceptos como la detección temprana, la prevención y la salud mental frente al concepto más utilizado de enfermedad mental.

Comenzamos nuestra sesión de concienciación recordando lo importante que es la detección temprana de la enfermedad mental partiendo de una octavilla de la organización inglesa Rethink Mental Illness (http://www.rethink.org, Figura 1). En la octavilla que empleamos dice: "Cuando tu coche se avería, puedes recibir ayuda en menos de 60 minutos. Cuando es tu mente la que se avería puede que la ayuda tarde 18 meses".

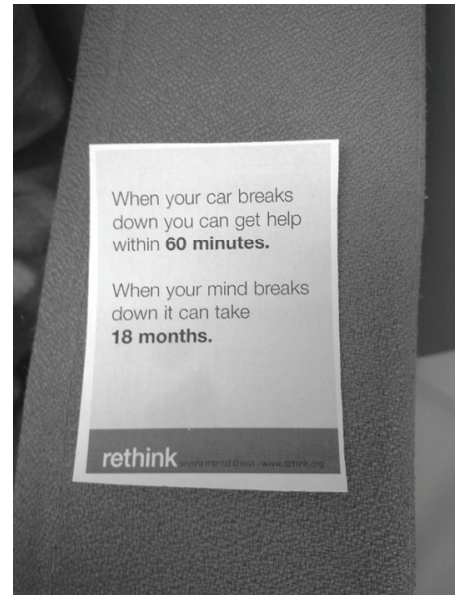

Figura 1. Campaña de concienciación para la detección precoz de la enfermedad mental de Rethink Mental Illness.

Esta tremenda demora tiene que ver con una incapacidad para detectar la enfermedad mental, no sólo de pacientes y familiares, sino también de los profesionales. Con una serie de vídeos muy breves repasamos lo que es prevención primaria, secundaria y terciaria.

Prevención terciaria es lo que en general se hace en los dispositivos sanitarios de salud mental que conocemos. El paciente con una patología definida acude para no tener recaídas o no empeorar. En la UME es lo que hacemos, recibimos personas con TMG y hacemos con ellos un programa de rehabilitación para evitar que tengan recaídas y para que vuelvan a tener un nivel de inserción social normalizado.

La prevención secundaria consiste en detectar lo antes posible un trastorno ya establecido y es en lo que incide la octavilla de la Figura 1. Para ilustrar este tema proyectamos para el grupo dos anuncios que se han emitido en televisión:

Una campaña de detección precoz en jóvenes de la Asociación Shift del Reino Unido (http://www.youtube.com/watch? $\mathrm{v}=\mathrm{Ju} 5 \mathrm{~d} 3 \mathrm{rW} 4 \mathrm{k} 1 \mathrm{o}$ ). El texto es éste: "Cada historia tiene dos caras. Uno de cada diez jóvenes padece un trastorno mental. No vivas de lo que crees. Conoce la verdad."

Una campaña de detección precoz en niños dentro de la campaña STARS for Children's Mental Health (http://www.youtube.com/watch?v=-ZHLyPgha4A). El texto es éste: "Conocemos muy bien las enfermedades infantiles habituales... pero algunas son difíciles de ver: cambios de humor, ansiedades, tristeza, hiperactividad pueden ser signos de un trastorno. La salud mental del niño es tan importante como su salud física. Aprenda más y ayude a que su hijo se sienta mejor por dentro y por fuera." 
Y finalmente, la prevención primaria consiste en algo muy lejano de las políticas de corto plazo actuales: prevenir de verdad, antes de que la enfermedad esté instaurada, mejorar realmente la salud mental de la población. La OMS tiene una publicación de 2010 que se titula: "La promoción de la salud mental en jóvenes - una inversión de futuro".10 Como toda inversión es algo que cuesta dinero, pero que sería muy rentable, eso sí, a largo plazo. Se proyectaron dos vídeos:

El de la escalofriante campaña australiana "Chidren see. Children do" de la Asociación para la prevención del maltrato infantil NAPCAM (http://www.youtube.com/watch? $\mathrm{v}=\mathrm{aANHqDUqQoI}$ ) en el que se ve a niños teniendo comportamientos agresivos copiando lo que hacen sus mayores.

Una campaña de promoción de la salud mental en el Reino Unido en la que una voz en off le dice a un adulto que se queda tirado en el sofá viendo la televisión (por desgracia, de esta campaña ya no queda rastro en Internet): "No puedes evitar sentirte mal a veces. Pero sí hay cosas que puedes hacer para sentirte mejor, cosas sencillas, como estar activo, mantenerte en contacto con los demás; incluso cambiar lo que comes... Sabes que hay cosas que pueden hacer que te sientas mejor. Así tú eliges."

Por último, proyectamos el vídeo de una campaña de la ONCE (http://www.youtube.com/watch?v=u8PDmtLFVqU) en el que se ve a Freud respondiendo a la siguiente pregunta: ¿qué es una persona sana?. La respuesta es muy escueta: "cualquier persona capaz de amar y trabajar."

La sesión psicoeducativa terminó con un ejercicio de escritura en el que se pidió a todos los participantes que escribieran también en una octavilla su propuesta para la promoción de la salud mental. Dimos unos minutos para que cada uno de los participantes pensase su propuesta y la escribiese. Luego leímos cada uno la nuestra y las pegamos en un ventanal de la sala de estar de la unidad donde estábamos reunidos (ver Figura 2).



Algunos de los textos fueron: Poder hacerme cargo de mí con mis grandezas y con mis dificultades Salir adelante pensando en ti Un futuro mejor es posible Ganas de vivir Ayuda y valentía para salir Salud mental $=$ disfrutar de la vida

Figura 2. Imagen de los textos escritos por los participantes en el taller conformando un tablón de propuestas en una ventana del centro

${ }^{10}$ WHO (2010). Mental Health Promotion in Young People - an Investment for the Future. Ginebra, World Health Organization. 


\section{TERCERA DINÁMICA: UNA DINÁMICA CORPORAL DE TODO EL GRUPO}

Nos planteábamos el empleo del propio cuerpo en la resolución de ansiedades vinculadas con el estigma en la enfermedad mental, con el empleo de técnicas psicodramáticas. 11

El cuerpo que nos constituye en personas forma parte esencial de la unidad psicosomática que es el ser humano. La vivencia corporal en la normalidad o la patología, constituye un elemento fundamental para conocer y abordar desde la antropológica y la psicológica. Conocer nuestro cuerpo, "habitarlo" es necesario para ayudar y ayudarnos. Afirma Alexander Lowen que lo más importante en las experiencias humanas es que encuentran su expresión no sólo en la función mentalpsíquica sino también en el cuerpo: en la postura, en patrones de reacción y también en inhibiciones de la movilidad, respiración y expresión, algo que observamos habitualmente en nuestros pacientes.12 Estos patrones corporales representan una estructura caracterológica que influye en la percepción física del yo, en la autoimagen $\mathrm{y}$ en los patrones básicos de intercambio con el medioambiente. Pensaba Lowen que nosotros suspendemos nuestros sentimientos e impulsos como resultado de una vuelta atrás en la expresión, y que esta regresión expresiva se convierte en una actitud habitual e inconsciente del cuerpo.

A través del cuerpo que juega, que se deja ir buscábamos la respuesta espontánea del grupo. Aprovechamos que el movimiento corporal supone a nivel psíquico un estímulo para las capacidades sensoriales y mentales, y desde el punto de vista social facilita las relaciones con el entorno y las personas. Si las primeras dinámicas se encuadraban en lo verbal, si en ellas el vehiculo principal eran la mente y la palabra y nuestra posición era sentada o de pie ; ahora el cuerpo y el movimiento se apoderaban del espacio y el grupo se expresaba. Recordemos a Bateson "Somos tanto una respuesta a los estímulos de la gente como un estímulo para su conducta".

El terapeuta ocupacional propuso un trabajo donde se borraban las fronteras de la palabra, para pasar a la acción y donde los cuerpos se encontraron sin reservas, sin fronteras. La consigna inicial fue muy sencilla: por turnos iríamos moviendo nuestro cuerpo y los demás nos imitarían. Así el enorme corro dio una vuelta donde todos, incluidos nuestros usuarios con mayores problemas de aislamiento y timidez participaron con normalidad. El ejercicio terminó de forma espontánea en un enorme abrazo que nos resultó muy agradable y reconfortante y nos acogió a todos.

${ }^{11}$ PAVLOVSKY E. Espacios y creatividad. Buenos Aires: Ayllu, 1985.
${ }^{12}$ LOWEN A. El lenguaje del cuerpo. Barcelona: Herder, 2000. 


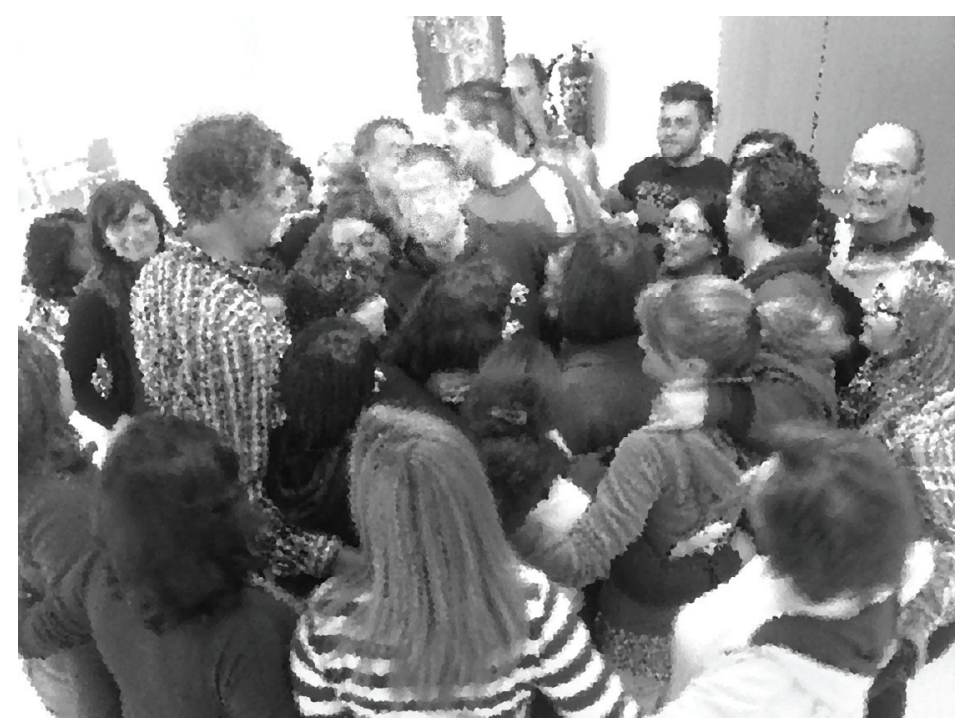

Figura 3

Figura 3. Final de la sesión de terapia-expresión corporal en la que todo el grupo de visitantes y personal y usuarios de la UME acabamos escenificando y compartiendo un enorme abrazo.

\section{CUARTA DINÁMICA: UNA SESIÓN DE PSICOEDUCACIÓN POR PARTE DE LOS USUARIOS}

Por último, después del gran abrazo que nos dimos y que todos recibimos del grupo, los usuarios hicieron una exposición de los grandes grupos de enfermedades que tienen cabida en el centro: esquizofrenia, trastornos de la personalidad, trastorno obsesivo-compulsivo etc., síntomas, causas, tratamiento. Su exposición fue clara y breve y se centró en las consecuencias del TMG y en todas las limitaciones que impone. Por último leyeron al grupo el artículo "La UME de Guadalajara", escrito por los usuarios de la UME y publicado en la revista del Centro de Rehabilitación Psicosocial y Laboral de Guadalajara (Figura 4). 


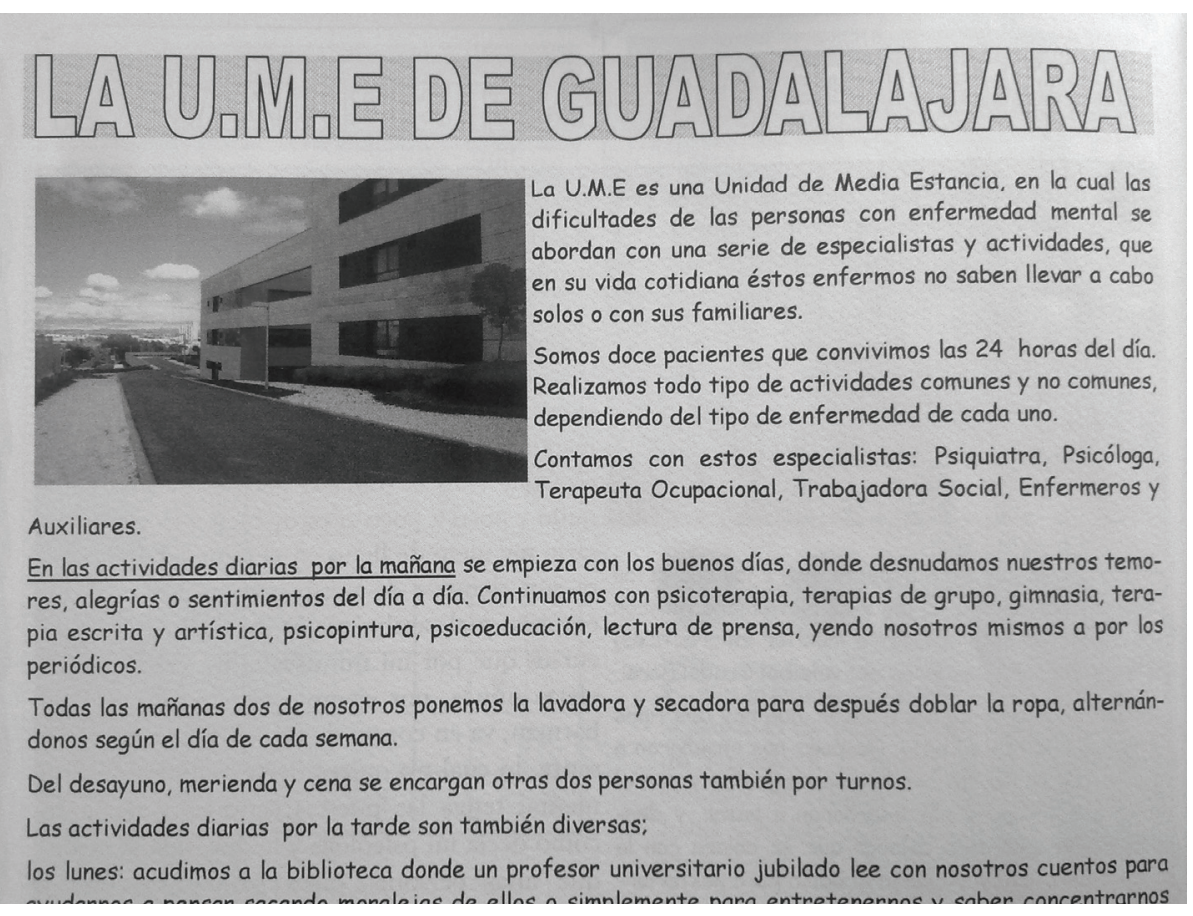

Figura 4

Figura 4. Artículo de los usuarios de la UME en la revista Tisana publicada por usuarios del Centro de Rehabilitación Psicosocial y Laboral de Guadalajara (CRPSL).

De la enfermedad a la persona, camino que recorremos con los pacientes cada día, en el proceso de empoderamiento de su salud, reconocer sus síntomas, ponerles nombres, reconocer el curso de sus enfermedades, los tratamientos, la importancia de su implicación en los mismos; ponerse delante de los otros,- los otros estudiantes-, para enseñarles sobre salud y enfermedad mental, supone una capacidad de exposición, aceptación de su trayectoria vital que sin duda resulta sanadora.13

Después del abrazo ya no nos volvimos a sentar. Permanecer de pie fue algo espontáneo que nos permitió estar más cercanos el resto de la visita (Figura 5).

${ }^{13}$ RODRÍGUEZ, A. Rehabilitación Psicosocial de Personas con Trastornos Mentales Crónicos. Madrid: Editorial Pirámide, 1997. 


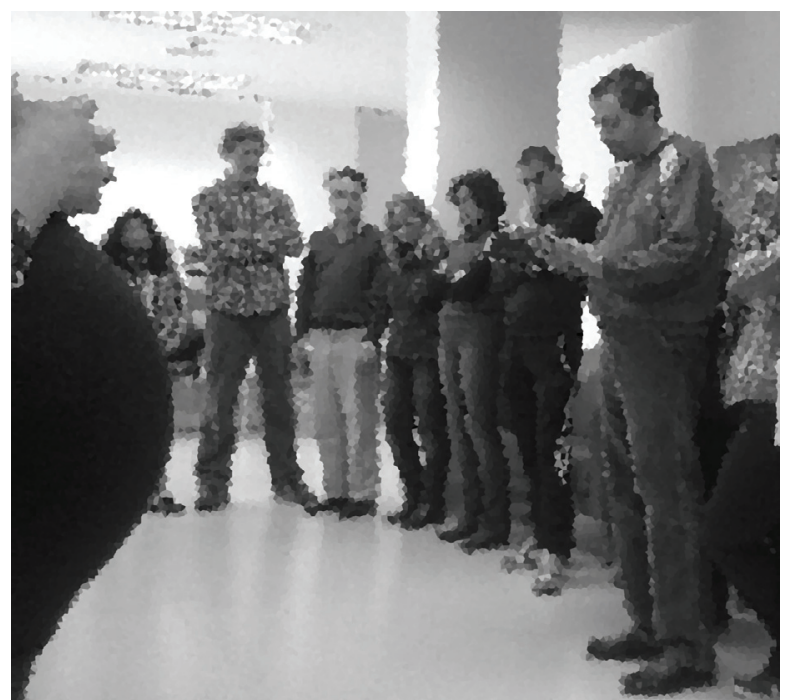

Figura 5.

Sesión de psicoeducación por parte de los usuarios.

Cuando terminó la sesión psicoeducativa por parte de los usuarios, hicimos una visita guiada por toda la unidad en la que los guías éramos tanto los terapeutas como los usuarios. Los visitantes preguntaban y les respondíamos unos u otros. Los anfitriones fuimos todos y durante un rato, los que durante las primeras dinámicas habían estado sentados juntos pudieron hablar de forma más abierta dejando atrás sus roles de visitantes o de usuarios o terapeutas de la UME.

\section{CONCLUSIONES Y REFLEXIONES}

De una oportunidad inesperada surgió una jornada que fue muy fructífera para nosotros, pacientes y profesionales, un equipo en el que todos sabemos el lugar que ocupamos en nuestra tarea común; y también para nuestros visitantes, como nos comunicaron posteriormente las tutoras.

Aprovechar cualquier experiencia para trabajar el estigma, el empoderamiento, y hacerlo desde la representación, el juego, desde un todo holístico donde el cuerpo vive, siente y hace sentir, acorta las distancias. Una representación en la que en algún momento hemos sido el otro, nos ayuda a comprender desde las emociones, no sólo desde el pensamiento. En este caso aprovechamos la experiencia para trabajar juntos desde diferentes disciplinas, terapia ocupacional, psiquiatría, psicología, aportando cada uno nuestros conocimientos que enriquecen el trabajo y nos nutren a nosotros y sobre todo a nuestros pacientes. Utilizando los medios a nuestro alcance, no muy sofisticados, no muy abundantes, estimulamos la parte sana de nuestros usuarios. 
Nuestra conclusión es que queda muchísimo por hacer en cuanto a combatir el estigma y promover la inclusión social de los pacientes con TMG y también para promover políticas preventivas a largo plazo. Pero también somos optimistas, porque cuando se da la oportunidad, tanto las personas con una enfermedad mental como los que en principio no las padecen estamos todos dispuestos a aprender y a cambiar. El estigma se pude combatir. La experiencia mereció la pena, con aportaciones de todos, con videos, debate, expresión corporal; actividades que por otra parte, realizamos muchos días, pero que en esta ocasión además contábamos con visitantes.

Aquella mañana la risa fue catártica. Viajamos desde aquel juego que nos colocaba a todos y a todas en tan diferentes lugares y que mostró a los que allí estábamos presentes lo prejuicioso de las etiquetas, hasta las reflexiones sobre salud y enfermedad. Reflexionamos sobre la importancia y la dificultad de la detección precoz de la enfermedad mental. Asistimos al empoderamiento de los pacientes en relación con su propio proceso. Con la dinámica de expresión corporal terminamos dando lugar a UN GRUPO, un nosotros, donde todos mezclados éramos capaces de compartir emociones y aceptarnos sin fronteras.

Cuando los estudiantes se marcharon nuestros usuarios se sentían vivos, despiertos, contentos de haber podido conectar con personas de su edad dejando de lado su enfermedad. Si la pudieron apartar esa mañana la podrán apartar más veces y más tiempo.

\section{REFERENCIAS BIBLIOGRÁFICAS}

LINQVIST P, ALLEBECK P. Schizophrenia and crime: a longitudinal follow-up of 644 shizophrenics in Stockholm. British Journal of Psychiatry 1990; 157:345350 .

LÓPEZ M. y LAVIANA M. La lucha contra el estigma y la discriminación en salud mental. Una estrategia compleja basada en la información disponible Rev. Asoc. Esp. Neuropsiq. 2008 v.28 n.1 Madrid.

GONZALEZ PARRA S, MOLINA JD, HUERTAS D. Violencia asociada con los trastornos esquizofrénicos. Archivos de Psiquiatría 2008, Núm. 2-8-Vol. 71-p. 109-127.

CORRIGAN P W, WATSON AC. Understanding the Impact of Stigma on People with Mental Illness. World Psychiatry, 2002, 1, 1, pp. 16-20.

LAVIANA M. La atención a las personas con Esquizofrenia y otros trastornos mentales graves desde los servicios públicos: una atención integral e integrada en un modelo comunitario. Apuntes de Psicología 2006, 24, 1-3, pp. 345-373.

WHO (2010). Mental Health Promotion in Young People - an Investment for the Future. Ginebra, World Health Organization.

PAVLOVSKY E. Espacios y creatividad. Buenos Aires: Ayllu, 1985. 
LOWEN A. El lenguaje del cuerpo. Barcelona: Herder, 2000.

RODRÍGUEZ, A. Rehabilitación Psicosocial de Personas con Trastornos Mentales Crónicos. Madrid: Editorial Pirámide, 1997.

WALKER, Leonore (1979): The Battered Woman. Harper \& Row. Nueva York 